\title{
The Alternative Mathematical Models of the World
}

\author{
Salahaddin Khalilov \\ Azerbaijan University
}

\begin{abstract}
The biggest insufficiency of the present mathematics is that it consists, in fact, of the eclectic mixture of two different models. In the first model, the world is taken as infinity and what is chosen as an element here is the unit. Everything is measured by the multiples of the unit. According to the second model, this world is in unity and its mathematical manifestation is the unit. The world is between zero and one. Everything here is measured by fraction. The simplified variants of the mathematical model of the world paves the way for the comparative analysis of its alternative philosophical interpretations.
\end{abstract}

Keywords: world models, zero, unit, infinite in finite, finite in infinite

\section{Introduction}

The leading principle in the majority of scientific teachings is the cumulativity. It is acted from the naive faith that by gathering knowledge about the separate events and things of the world, we can get knowledge about the world as a whole. In fact, it should be moved not from parts to the whole, but from the whole to parts.

When the world is accepted as a unit, then its mathematical model also changes. That is to say, our approach to the world could be understood not as an approach to infinite, but as an approach to the unit. In this case, all other events (and knowledge about them) are expressed as the parts of the unit, that is, as fractional numbers.

Depending on how to explain the unit and zero, it is also possible to establish two different models of the world.

\section{Infinite in Finite and Finite in Infinite}

Any part of this world is endless and infinite. Is there, then, anything finite in this world?

It is possible to put the question "Can infinity be included in the finite" as follows: "Is there an idea that includes infinity in this material world?" A very important problem is put forward by putting the question in this way; the possibility of the idea's being an element of man's world (consciousness) is reminded here. Every material thing is indefinite for human consciousness. What the human being can recognize from the material world is only the prototype of the things which has pre-existed in the mind; and what he does not recognize will stay in the background as indefiniteness, to wit, as infinity or darkness. In fact, man's own world is not clear to himself. Everything in darkness is indefinite. The illumination of any idea can be understood also as its coming

Salahaddin Khalilov, professor of philosophy at Azerbaijan University, head of the East-West Research Center, Editor-in-Chief of the international journal Felsefe ve Sosial-siyasi Elmler (Philosophy and Social-Political Sciences), Department of Philosophy, Azerbaijan University, Azerbaijan; main research fields: Philosophy of Science, Science of Science, Philosophical Comparativism, Phenomenology, Philosophical Aspects of Eastern and Western Civilizations, Philosophy of Abu Turkhan, and Cognitive Theory. Email: ssx@azun.baku.az. 
to focal length.

The teachings and mathematical models, which take the world as infinity, choose the unit as element. Everything is measured by the multiples of the unit. The atomistic teachings are also based on this very idea. Therefore, there is a smallest indivisible final limit of this world which has not yet been broken; and this limit is also finite. The most adequate expression of it in the number plane is the unit. The atom of natural numbers is also the unit. All numbers consist of the different multiples of this atom and this unit. It is true that, in parallel to it, the notion of fraction is also used in mathematics. Namely, it turns out that beside natural numbers, there are also fractions. However, this kind of approach arises from mixing two mathematical systems and two different models as well as from their eclectic unity. This, in fact, is the biggest insufficiency of the present mathematics. Due to this turmoil and confusion, it has not yet been possible to create a simple mathematical image of the world. Thus, if the atom of the physical reality is accepted as adequate to the notion of the unit in mathematics, then the existence of fractions becomes impossible because the atom is indivisible according to its definition.

One of the two ends of the world becomes finite and another infinite when this type of model is established. That is to say, this model includes the infinitude, but it does not however include the infinitesimal because there is not a smaller one than the unit. However, it is possible that there can be a completely opposite model to it. The world itself is finite according to this model, in other words, it is the unit; and infinity is within it. In this case, what exists is the infinitesimal, not the infinitude. What is paradoxical here is that the infinitudeness finds room in the finite, that is, in the unit, or it is crammed into it.

It is not indeed so easy to give its philosophic-ontological explanation. This mathematical model of the world is very simple in turn; and the world is between zero and one. Everything in this world (the world of multiplicity in philosophy) is measured by fraction. The change is made not in the numerator of fraction but in its denominator. Numbers, in turn, serve to show how many pieces the unit is divided into: Every element is $1 / \mathrm{n}$, where $\mathrm{n}$ is a set of natural numbers $(0<\mathrm{n}<\infty)$.

This world is in unity as a whole and its mathematical manifestation is the unit. It is possible to approach the unit, whereas it is not possible to reach it. This thesis also reminds us of the thesis which claims that "it is possible to successively approach the truth, but it is impossible to reach the ultimate truth." Beauty, Good, etc., also are visible as an ideal on the horizon.

The main point is the differentiation between the particular and the general as well as between thing and idea. Aristotle mainly used the term of species. That is to say, in spite of their specificity, each type is expressed with the notion of the unit (it also reminds us of Russell's logical atomism). What is chosen as particular or what is specially differentiated as general-it all depends on what structural level of the world's model or map is drawn by a man, is considered. For example, in agricultural activities, it is possible to take as a basis not the fruit, and not the apple, but a specific kind of apple, and next specializations can be conducted within that type. If we speak however on a large scale, it could be said that the kinds of apple are not important to us and they can be taken as the specific conditions of the universal apple. In this very stairs, it should be taken into consideration on which step we stop for viewing the world, that is, we should pay attention to the structural level of hierarchic order.

Thus, depending on the models of the world as well as on which scale and which focal distance we review it from, units change. However, every approach and measuring system have their own unit and everything starts from how the unit is chosen. The next architectonics, as a whole, is based on it. 
However, if every kind has its own unit, then to what extent is it important that how many times these kinds of thing and events are repeated? To make it clear, it could be claimed that although there are many material manifestations of the kind, none of them is original, that is, none of them is exactly unit. The unit always consists of idea and its material manifestations, in turn, depends on where and which things it is reflected and copied in. Mathematics (quantity), in fact, acts from the numbers of copies. Mathematics does not exist in the world of essence. What exist there are only units and they are all equal in rights. The repetition of one essence and one idea twice also is not possible just because there is no time-space continuum there. Namely, if an idea or essence exists, it exists everywhere and every time and there is no need to a second one, in other words, it is impossible even to imagine a second one. Ideas always exist alone and purely; time-space continuum is for expressing the multiplicity and different combinations (structuralizations) of copies. In this sense, numbers as well as their combinations, that is, mathematics as a whole belong to the material world, or more precisely, mathematics is a bridge and means of transformation between the material world and the world of ideas.

Every structure level, in fact, corresponds to a sphere of the world. There is not an uninterrupted transition between these spheres; there is only a discrete transition. Just like electron shells in N. Bohr's atomic model, which, each of them being one sphere and one layer, can only discretely pass to the next layer, and although the world is uninterrupted at a certain level of structure, transition to another level of structure becomes possible only discretely by changing the paradigm.

Thus, there is one general or idea that the unit is on the basis of each type. However, due to a higher generalization, these units themselves, in the higher stages of hierarchy, are transformed into manifestations, the particular states of a more general, and the copies of the unit which stands in a higher layer. Then it is not important that what type is of concern as well as the manifestations of an idea or an essence are no longer compared with each other by quality, on the contrary, they are compared by quantity; as well as it is elucidated that how many manifestations, events, and things are of concern, or how many multiples of its unit it corresponds to. Here it becomes necessary to explain the measurement unit of the physical parameter and it is also necessary to elucidate what part of this unit the concrete physical object keeps, and only in this case mathematics steps in. Or, when the relation among two events, properties, and physical quantities is determined, each of them are represented with their appropriate multiples or they are represented in a form that has been changed as the result of a mathematical operation. Mathematical relation, equation, and formula cannot belong to a concrete manifestation and a relation between material objects. First of all, it is necessary for this to idealize those material objects - model and symbolize, or in other words, it is important here to precisely discover the ideas that they carry.

It is possible to measure different properties and characteristics of any material object. However, the constant relation and regularity, which can be expressed by a formula, cannot belong to a specific material object, but to this or other idea and the general that it carries, or more precisely, to the relation between ideas, and to their structure. For example, it would be more correct not to speak about the attraction or repulsion of an object, which has electrical charge, by another charged object, but about these electrical charges' attracting or repulsing each other. Or, the mathematical regularity in gravitation does not express the relation between two objects, but it expresses the relation between two masses. In this case, we do not pay attention to the size, form, and other peculiarities of objects, but to their mass, and this mass should not be considered as the mass smeared out in a volume, but to a certain point in space. Namely, if the first point mass is $\mathrm{m}_{1}$ and the second point mass 
is $\mathrm{m}_{2}$, there will be a gravitational force between them which is directly proportional to the product of the two masses: $P=\gamma m_{1} m_{2} / r^{2}$. That is to say, the constant mathematical relation-formula, does not express the relation between any specific objects, but the relation between abstract universals. Both the mass and length have their own unit. Their symbolic sign, in turn, shows the multiples of that unit. For example, when we speak about $\mathrm{m}_{1}$, we mean the number of the multiples of the mass unit. In other words, $\mathrm{m}_{1}$ is only a number in mathematics. Namely, the gravity formula that we mentioned above simply expresses the product of several numbers in numerator, and an ordinary calculation operation, which is considered as the square of a number in denominator. What makes the indicator of the physical reality is the multiple of what unit is these numbers. Then the carriers of the meaning are not multiples and numbers, but units. Therefore, the unit is the example of the kind and essence.

What carries the meaning is not multiples and numbers but units, because the unit is the symbol of species (types) and essence. It becomes necessary here to differentiate between "the unit" and the notion of "one." It is not a coincidence that for elucidating whether the unit has quality characteristics or not, Gottlob Frege (1960, 39) also suggests distinguishing between the unit and the notion of one.

\section{Zero and the Unit-The Internal Dynamics of Zero}

Is the beginning unit? Or, does everything start from zero? As the unit has its opposite, zero does not have an opposite or it is equal to itself. That is to say, it is possible to accept zero as emptiness and a notion that has not any content as well as it could be viewed as a joining of positive and negative unities (opposites). From this standpoint, zero seems to be more fundamental than the unit. This is in line with the statements of the Bible: "Very truly I tell you, unless a kernel of wheat falls to the ground and dies, it remains only a single seed. But if it dies, it produces many seeds" (Bible John, 12: 24).

Modern scientific studies show that along with matter, there is also antimatter and the annihilation process happens when they contact each other, that is, both of them "disappear." The contrary process however is also possible, that is, the creation of two positive and negative signed particles (for example, electron and positron) from this non-existence and zero, in a certain condition, is also possible. This, in turn, shows that the form of existence, which is accepted as nothingness, non-existence, and zero, is in fact a more complex being. Just as atoms, which are neutral (because positive and negative charges balance each other), the positive and negative directed deviations in the social life, for example, good and evil, also neutralize each other. The normal state, which includes both good and evil, does not arrest attention and what arrests attention is only the good or the evil, which comes to the forefront in a certain situation and breaks the balance. Mathematically expressed, 1 and -1 have a certain value, but 0 and its multiples have no value. Whereas when 0 decomposes, it becomes equal to the sum of 1 and -1 , that is, it turns out that it is included in both of them.

The results of our study and the modern thinking in general require the changing of the attitude towards zero. Zero is not only a symbol of emptiness, but also a symbol of completeness, balance, and neutrality. In this case, zero competes even with the unit. For the reason that the traditional standpoint considers the unit as a symbol of unity and completeness, it has captured one of the shades of meaning of zero. It is worth here reminding that zero also means silence and rest. Silence in fact is the absence of sound and rest, in turn, is the absence of motion. However, the absences of other qualities like mercy, love, generosity, etc., are mostly expressed not with the zero point and the neutral situation, but with the existence of opposite qualities, for example, with cruelty, hatred, stinginess, etc.. These are however the other poles of those qualities. Then how is 
the zero point defined? It is mostly accepted as a beginning or transition point, that is, it is accepted neither as mercy nor as cruelty, neither as love nor as hatred, and neither as generosity nor as stinginess. Nevertheless, it becomes possible only when the model that we have established is the one-dimensional space. Namely, the change happens between -1 and +1 and 0 is only a transition point here. However, even within this model, we can accept zero not only as the absence of positive and negative qualities, but as the unity and sum of their states which are equal in value but opposite in direction. It is called dynamic balance. Even sometimes not some love or some hatred but the culmination of both of them can be together. It is true that balance can often be disordered in this case as well as the leaps from one pole to another can also be possible. However, the entire panorama can only be expressed by means of the dynamic zero. We can also describe events with two-dimensional and three-dimensional spaces. In this case, any state of quality should be taken as a vector quantity. Then together with the opposite diametric positions of these qualities to each other, their states under different angles could also be taken into consideration. The state of zero of this quality can become possible only when the net vector is equal to zero. That is to say, the state of zero rarely becomes possible in reality or it is only an idealization. For us, it is possible to describe the real situation as the fluctuations around zero and give its mathematical expression.

We have cited a number of examples from spiritual life. The application of mathematics in this field is still next to nothing. Mathematics is mainly used in the explanations of physical events. For this reason, it is worth here paying attention to the more comprehensive states like warmth and coldness, and positive and negative electrical charges.

For the reason that heat is the final indicator of statistical event, it is slightly difficult to apply the vector quantity model to this area. If we finally move on to the motion trajectory of separate particles and try to determine the final temperature by summing up the vectors that indicate the motion of these particles, the problem will become more complicated. Statistical methods are applied to escape from this very complication.

Here a very important philosophical conviction appears; it turns out that the rest state point is pithier and more complex than the motion point. Namely, motion only covers the deviations from the state of equilibrium and the neutral situation, and in turn, the issues are mainly concrete and their calculation is possible, because the state of balance (chaos, homogeneous environment, and the equality of the net vector to 0 ) is accepted as a reference system or a beginning, or, expressing it with mathematical symbolism, zero state. However, the internal structure of the state of balance is more complex and the internal processes are either the only reasons for these motions or together with outside impacts.

One of the important points belongs to the dialectics of unity (wahda) and multiplicity (kasra). Namely, approaching from ontological context and acting from the essence, the unit can be taken as the only being. The universal unity or the unity of the world is understood so that everything exists within it and is a part of its harmony and there is nothing outside it. What is the multiplicity then? How, in which points and in relation to which things does diversity appear?

If we act from the second mathematical model of the world, that is, if we put the world in the space between 0 and 1, diversity, in turn, will naturally appear within this space. The world will be then filled by the diversity that includes the one-half, one-third, one-hundred, etc., of the unit till infinitesimal manifestations are as well as incomplete beings. However, as we mentioned above, if we take the hierarchic structure into consideration, every circumference and every sphere will be able to possess their own units and decimal 
systems; more precisely, the distance between 0 and 1, by being divided firstly into 10, then 100, 1000 and other parts, can create a conditional stratification and a multistorey system. The division in this model, however, is realized in a different direction. The unit manifests itself from different perspectives as different kinds, and every cross-section has its own internal gradation.

\section{The Mixed Model and the Polysemy of the Unit}

The present mathematics is established not in the first or second model of the world, but in the mixed form. That is to say, it is intended here that the world is situated not between 0 and 1 or 1 and $\infty$, but between 0 and $\infty$ or even between $-\infty$ and $+\infty$. The idea of the discrete structure of the world has been instinctly (blindly) abandoned and mathematics has developed on the idea of continuity for a long period of time. This approach has become so ordinary that not only mathematicians do not attach great importance to dividing the unit, but also they are not aware of what kind of step they make. In particular, the emergence of the differential and integral calculus has strengthened this position. It is true that, also, sometimes unconsciously, some models are built on the basis of the idea of discreteness. However, it is not given any sufficient philosophical explanation of these transitions. In this context, the study of the internal relation between functional analysis and higher algebra remains as one of the actual duties.

The unit in mathematics moves out from its philosophic-ontological meaning and acts as an element of all possible structural variants. Unlike the first model, the unit here does not express the entire world and the wholeness of this world; on the contrary, it acts as an element and indivisible basis of all things and types. Each type of wholeness and each relatively completed system can be accepted as an element when it moves on to another scale and another level of structure. For this reason, every type has its own unit. On the other hand, because all the same kind of material manifestations and singles are expressed by the unit, it emerges here quantitative index - the notion of number.

Indeed, it can be many units in mathematics. For example, every human being separately or many gathered people as well as the whole society and humanity can be taken as a unit. Although a great number of people are gathered in every group, class, political party, and social association, their sum is equal to the unit. Without going into the details of the teaching on groups, pluralities, and matrices, we would like to direct the attention to the possibility of taking every group (plurality, matrix) as unit and comparing it with another unit (group). That is to say, in one sense, it is possible to view the relation between two groups as a relation between two units. However, now we are interested in another situation. We are going to analyze the relation of the unit with plurality in the example of man and group. For instance, a teacher speaks and his/her words are addressed to each of 25 students in the lecturehall. All students, in turn, without having any relation between themselves, listen to the same person - the teacher. Or, a singer sings on stage and at the same time, he/she is in one-sided contact with thousands of spectators.

We can give other examples of the simultaneous relation of the unit with plurality. Our aim is to pay attention to the unity point of plurality and variety. We would like once again to emphasize that these kinds of relation systems are deprived of feedback. Namely, what is possible is only the relation of the unit to another unit. Plurality is in contact with the unit (subject) only as the object. That is to say, this relation is only passive and one-sided. Each of many people can direct attention to the same direction, but not all of them can be the active side, because they become a side all together. The mathematical model of this kind of situation, for instance, can be described in the form of relations of the central point and the different points of the circle. 
If we transfer the above-mentioned points to philosophical problems, it should be taken into consideration that only its own idea and essence could be accepted as unit for every kind. The idea here is the unit. All the objects, which manifest this idea and essence in themselves, will become a passive plurality—variety.

\section{Units and the Absolute Unit}

We focus attention on the notion of the unit so that, in addition to any other thing, we could talk about the unity of the world as a whole or the unit world. For example, a building is being under construction and it is considered completed when it becomes fully adequate to its project. For this reason, always something is insufficient, that is, realization and reality are imperfect comparing with idea. It is not a coincidence that only idea is considered perfect by Plato. In other words, only idea can be a unit; “... he who thinks of number will be able to think of the unity and plurality apart; number then will be this - a unit and plurality, or the one and the unequal" (Aristotle 2001, 923).

However, to imagine the kind, essence, concept, and idea of different things in the end as a standard, ideal and unit is one thing, and to imagine the world in whole as a harmonious, logical, complete, and perfect unit is a quite different thing. Namely, the existence of every species becomes possible in the places and environments of other species. In order to achieve the level of perfection, each kind struggles with its environment, and as large this environment is, there is more likely an approach to perfection. So, anything taken separately only by adapting to the whole world context and, in fact, to the harmony of the whole world, being free from all contradictions can reach the perfection. In other words, the wholeness and perfection are better not to be against the world, but in unity with it.

According to conventional notions, the world is infinite and it appears that, it would be marked in mathematics with the sign of infinity. However, for the mathematical expression of the world, we offer just the unit.

It seems that, there is a need to enter a new concept - the concept of the absolute unit. All the other units are units—standards only for their types (community, nation, etc.), but the absolute unit includes two different qualities. First of all, it is unique and there is nothing out of its will. Secondly, it represents everything that it includes, i.e., it is the most general of all generals. For example, the Sun is single. However, it is not general. Despite the fact that it insolates the objects and events in the world with its rays by lighting them, this process is interruptible and occurs in a limited circle. Nevertheless, this quality of the sun has become, at different times, the cause for people to worship and divinify it. People's consciousness has always been in quest of a more universal one that has the quality of generalization.

One of the most paradoxical problems in philosophy shows itself in the dialectics of the unit and general. One of the most difficult points for the cognition is to understand the difference between the general and total. The total appears from the sum of singles. It is called a common factor in mathematics. The "one" is the most "universal factor" for all numbers. From the other hand for treating the sum as unit, all the summands in advance have to be fractions of the same unit, i.e., $a_{1}+a_{2}+\ldots+a_{n}=1$.

What is paradox is that, how the unit as a sum can be the same with the unit as a common factor.

However, for the reason that mathematicians do not consider the special burden of the unit and the difference of a unit from another one, they easily compare different weighted numbers with each other. Whitehead writes: 
But we are entirely abstracting from any consideration of any particular entities, or even of any particular sorts of entities, which go to make up the membership of either of the two groups. We are merely thinking of those relationships between those two groups which are entirely independent of the individual essences of any of the members of either group. (Whitehead 1925, 29)

Then Whitehead continues his view: "But the first man who noticed the analogy between a group of seven fishes and a group of seven days made a notable advance in the history of thought. He was the first man who entertained a concept belonging to the science of pure mathematics" (Whitehead 1925, 29). Surely, the greatest mathematicians explain the nature of their science in this way. However, how one can agree with this? It seems mathematicians are too keen on the abstraction, so they equate all units. The analysis made by us shows that units are different from each other.

Units are the same only within a certain type. While refusing to differentiate the unit that belongs to two different types, mathematics goes so far in abstraction that it becomes much farther from reality.

For mathematicians, the unit is as colorless, soulless, and lifeless as in other numbers. But the only means that connects mathematics to the real world and makes it worldly is taking into account the spirit and nature of the unit. Willard Quine tries to coordinate a geometric object with a physical object in his work Word and Object:

Thus we may fairly say that the question of the nature of the geometrical objects is, like the question of the nature of the elementary particles of physics, a question of physical theory. Granted, laboratory data only incline and do not constrain us in our geometrizing; but likewise they only incline and do not constrain us in our invention of a physical theory. (Quine 1960, 252)

"For, we may think of points, curves, and geometrical surfaces as ideally small particles, ideally slender wires, and ideally thin sheets" (Quine 1960, 251).

The philosopher concludes that, in this sense, geometry does not differ from physics so much. However, when the physical object actually loses its certainty, materiality (and thus non-certainty), and multiplicity, as well as when it is expressed by means of a number or a finite number of numbers, it becomes geometric, too. When the physical world becomes simplified at the highest abstraction level, it is transformed into geometry, and when geometry becomes simplified, it is also transformed into numbers.

But what is the "geometrical object?" What kind of necessity has made it necessary, and what kind of connection has it with numbers?

Numbers and scalar quantities are for the one-dimensional world. One number is enough for characterizing the rectilinear uniform motion. In fact, there is no need to know the beginning of coordinates, because, in this case, all the points can be taken as equivalents. There is only the distance (length) in one-dimensional space that is measured by one number.

In two-dimensional space, each point is determined by two points, in $n$-dimensional space by $n$-number. The transition from one-dimensional space into two-dimensional space is possible due to time. Because the rectilinear motion is cut in any point and goes to another direction. Continuing in a certain period of time in the same direction, it is cut once again and the direction changes and so on.

There is no time at the rest state. Space consists of a point and it is defined by zero. Although one number is enough while crossing from the point to the line in order to describe a constant speed rectilinear motion, there occurs a need in a new quantity either in speed or direction changing. In other words, time factors as well as new directions have to be taken into account. 
In a word, there is a need in vector quantities for taking into account the factor of time and space and for their description. The transition from scalar quantity into vector quantity stands on the basis of the transition from the world of numbers into the world of geometry.

The number of the dimensions of space is usually explained by the transition from the point to straight line, from straight line to plane, and from the plane to volume. However, what is important for the physical reality is not the space at rest, but the characterisation of motion. Therefore, the quantities expressing the change of direction and speed must be considered as the extra dimensions of space.

\section{Mathematical Unit and Physical Unit}

According to Plato, the whole idea is one, and yet, being one, and it is in each one of the many. Actually, we deal with not "various things," but things belonging to the same kind. Each of those things is one, i.e., they are determined in special-temporal space and they are non-repeatable. They are classified according to a main attribute, because in some cases extreme conditions are not important.

There are two terminological ambiguities, which bring to confusing being used in physics, mathematics, and philosophy. Firstly, notions "single," "one," "unit," and "unity," in spite of their similarity, have different meanings. They are more than different, and they are opposite poles. So "single" belongs to a material worldthe world of diversity, and represents separate things. The conception "one" or "unit" does not express separate things, but totality. The notion "physical unit" used in physics also indicates not diversity, but identity (sameness) or community (generality). Therefore, unit of mass, unit of length, etc., are common for all things. Things having various lengths differ from each other by multiple of the same unit, i.e., it is that how many times they are more or less of the unit.

It applies to the "quantity" notion, too. Actually this notion is opposite to the notion of "quality" and signifies amount and number. It is a bit strange that some properties and qualities in physics are expressed by not "quality" notion, but (physical) "quantity" one. In reality, "physical quantity" is not quantity; most likely it is a property or quality. For example, mass of a body is a property or quality state which is different from its volume, color, or temperature. A quantity (amount) is the indicator (index) of multiplicity of the "physical quantity." One "physical quantity" differs from other one not by amount, but by the nature of the "physical quantity," or peculiarity of its quality state, i.e., taking into consideration the difference of one unit from other unit, we see that the rest distinction appears in amount difference of the same unit.

Thus diversity itself appears in two types (ways): diversity connected with amount of matter (material) and diversity originated by the difference of idea itself.

Each kind and quality state has its own unit. Traditionally intertype (interspecific) diversity is measured by multiples of the unit and the multiple factor (number) may infinitely increase.

$1<\mathrm{x}<\infty$

That is a traditional model.

In the model proposed by us, a "physical quantity" varies between 0 and 1 . But number of types (kinds) is numerous. So each type is distributed by its amount between 0 and 1 . Therefore, we can describe world's model as a circumference of radius equal to 1 . Every point on the circumference will be unit of the corresponding type (kind). Its quantity variation may be represented as a location on the radius. If we mark the centre of the circle as 0 , that internal variety of types can be showed along the radius between 0 and 1 .

$0<\mathrm{x}<1$ 
According to this model, both intertype diversity and diversity of types are represented in the scale of the circle.

In the Middle Ages Islamic philosophy (for example, in Suhrawardi), size of a sense image, gestalt, or phenomenon is neglected. To be more exact, according to that philosophy quality, image-form, or type etc., lacks of quantity sign. For example, according to Suhrawardi, size of elephant and ant in imagination is indistinguishable. More precisely put gestalt and form, function of the thing occurs importance. Ants may differ in size, but all of them are ants. Else in spite of number of ants, all of them are represented by the unit notion of "ant." A picture is also a symbol and its size is a secondary question. In point of fact, the unit is imagephenomenon of "ant." According to Plato, the unit is namely an idea. Parmenides asks Socrates: "Then do you think that the whole idea is one, and yet, being one, is in each one of the many" (Plato 1997, 365) and Socrates confirms that thought. According to Socrates, idea is located in thought, soul, and spirit.

\section{Unity and Diversity}

Aristotle says: “... What is one in account is several in sensation” (Aristotle 1998, 22). Thus, unity is a quality specified only to the idea. The multiplicity (also divisibility, incompleteness) and diversity are related to material manifestations. We encounter a similar approach in the Ibn Arabi's teachings (Ibn Arabi 1946, 106) of al-a'yan al-thabitah (perennial archetypes). It is interesting that only unity is accepted as a true being in Medieval Islamic philosophy, and multiplicity, in turn, is as a mortal world. It is true that, what is of concern in Islamic teachings is only one unity and one unit-God. Ascribing the unit to every structural level in the world's architectonics, Abu Turkhan's philosophy paves the way for the implementation of mathematics. However, as many species there are, so many units there are. And the number of these units decreases every time when rising up to the highest abstraction level. Finally, all units become united in the same unit. Although the relation system with this unit resembles the model of the above-mentioned examples, they are not, in fact, the same.

It is true that other units including human beings form multiplicity and they are in a passive relationship with the unit. However, the elements of multiplicity stay here not just as audience but in a certain activity.

Therefore, this attitude resembles rather a conductor's leading the orchestra. An ordinary man just listens to a final and syncretic sound and acts from a common impression. Namely, the evaluation is subjective as well as it does not penetrate the object and cannot see it from inside. The conductor, in turn, controls both the music in general and individual performances. If the composer does not hear complex sound differentially, he can neither compose nor copy the notes. An artist also differs from ordinary people by features that he can see each color separately and imagine their various interaction combinations. An ordinary man only sees a general and final color and the world of colors is not open to him.

An ordinary man can do only single work at the same time: if his hands have got "a command" to do a single work, he cannot do any other work. There are few people who can do two different works by both hands separately at the same time. Such talent, for example, is demonstrated in circuses. In other words, it can only become possible when the hand's certain movements become automatized. Namely, only a certain movement could be leaded by active thinking.

The most important is that man can think only about the same theme at the same time. Man's ability to think about different issues is only a potential opportunity. Various events may happen at the same time. Man's attention however cannot be directed to all these events. The attention is concrete and depends on what thinking 
is focused on. The focal point is, in fact, spatial concreteness (When observation is carried out in huge space, then attention becomes weaker. When space becomes narrower, the attention-paid object comes into the focus distance and opens with all details). As the human being can direct his/her attention to the events only in turn, he/she can only revive them in his mind in turn. For us, the causality occurs from this very condition. In fact, before any event, so many events happen in the world that it becomes uneasy to determine what does the event, which we direct our attention to, and which arises from. The human beings make their own judgments only in the context of previously observed events. While, a great deal of events that stay beyond attention can also take part in conditioning results. However, the human beings build a model only within the limits of his knowledge and explain everything within the framework of this model.

One of the most important qualities that characterize God in Islamic philosophy is the potential to control all human beings and events at the same time. Only due to this quality, the whole world can become entire and harmonious. Finally, the victory of justice also becomes possible.

\section{Thinking Combinatorics}

The demand of connecting different empirical facts with one another as well as bringing them to a common denominator and commenting them within the context of same terminology, in fact, makes experiment dependent on theory. Namely, if the principles which are common for large-scale events are not definite, it becomes impossible to determine the scientific value of any fact and correctly place it in the system of scientific knowledge. Entering small-scale scientific teachings into the structures of larger-scale teachings is not as simple as it seems at first glance. The theses, which cannot be placed within a big theory, cannot yet be accepted as scientific theses. The establishment of a theory cannot be understood as a collection of known scientific theses and empirical facts. First of all, there should be a generalizing idea. The unification of existing scientific knowledge around this idea should also provide a number of conditions. The internal integrity of a theory, its simplicity, symmetry, etc., as well as the principle of parsimonious thought could be given as examples for these conditions. Einstein mentions that one of the main conditions in the process of finding the theoretical bases of different scientific fields is the condition of the minimality of participant notions and fundamental relations. If notions and relations in different fields can be logically taken from these theoretical bases, it means that the theory has been correctly established (Einstein 1940, 487).

If the world was constructed from small bricks (atoms), each brick could have been taken as a unit and all things and events in the world would be measured by its multiples.

Leibnitz held the following view "the best operation method of reason is that it reveals certain notions for itself and thus infinite numbers of other notions consecutively appear from them, just like it is possible to get all other numbers from several numbers." Leibnitz, still at that time with a great sagacity, noted that numbers are expressed by means of the unit (1) and nothingness (0). He explained the problem of existence and non-existence, in a certain meaning, by means of mathematical language. Eco used those ideas of Leibniz as examples to explain linguistic structuralism.

By taking notions as the atoms of thinking, Bertrand Russell brings forward the theory of logical atomism. The gist of this idea is that all texts, theories, and great complicated linguistic systems are based on notions and words. It is possible to create quite different combinations and structures from same words. Although the main idea of each text depends upon this very structure, its establishment as well as the expression of the opinion is guided, in the last instance, by words, notions, and their meanings. If notions and terms are correctly 
determined, the text formed by using them will enable them to become closer to the truth. In fact, the establishment of the text or the elaboration of any theoretical system is realized by means of logic and mathematics.

There are two sides participating in the formation of complex thinking systems: elements and structure. Notions act as element (atom) and logical constructions act as structure within the context of thinking. However, like in physics, the most difficult problem here also is that to what extent the nodes or notions taken as atoms are indivisible, constant, and ambiguous. The difference between mathematical and physical systems is that atoms within the first one are completely symbolic; in other words, for the reason that their elementariness is taken as a primary condition, it does not appear any indeterminacy in the next process, or more precisely, it is required not to appear.

The mathematical atom is the unit. For the reason that we cannot provide the identity of apples, when we talk about five apples in the material world, their values are not univocal. Namely, these five apples can be different from other five apples in a number of their indicators. For this reason, we do not talk in mathematics about a concrete object, for instance, about an apple, but about a conditional consent, for example, about the object "a." This object, in any condition, is equal to itself and cannot have any other sign, because it does not simply express any sign. The number 5 , in turn, shows that this "a" is repeated or multiplied five times. If we take as an example not "a," which is more abstract and symbolic, but a symbol, which indicates a physical quantity, for instance, " $m$ " (mass of object), although m's quantity characteristics will remain indeterminate, its definiteness, as a notion, will depend upon the definiteness of physical quantity that it indicates, that is, upon the mass of object.

Each of the three symbolic signs in the formula " $\mathrm{f}=$ ma" indicates a certain physical quantity (force, mass, and acceleration). If it becomes possible to exactly measure acceleration and mass, that is, if it is possible to express them mathematically with numbers, we can find the exact indicator of force expressed with numbers. In fact, the formula in question indicates the permanent relation between these three physical quantities. But if we doubt about the meaning of any physical quantity and make some corrections to it, this formula also will lose its importance. Alfred North Whitehead writes:

The point of mathematics is that in it we have always got rid of the particular instance, and even of any particular sorts of entities. So that for example, no mathematical truths apply merely to fish, or merely to stones, or merely to colors. So long as you are dealing with pure mathematics, you are in the realm of complete and absolute abstraction. (Whitehead 1925, 30)

The set of all natural numbers in mathematics is the example of such kind of world; the set, which starts from 1 and continues without reaching infinity, as well as can never include the world as a whole and at best can only reflect any big part of it.

If the world does not have any element, that is, if everything that seems to us as a whole is, in fact, the sum of parts or the fraction of an integer, that conditional element cannot be taken as a unit. Everything is a part and only the entire world is the unit as a whole. All that we see and know in this world are the parts of this unit. We can only conditionally accept them as the whole and unit.

If we have accepted anything conditionally as 1 , its twofold, tenfold, or million-fold is not of importance. The idea is the unit and there can be many copies of it. The essence does not depend on the number of a thing, but on the internal structure and nature of this thing (The nature of a thing, in turn, has already been completed at the distance between 0 and 1 ). 
The study and activity is carried out in two directions. The first is the definition of the phenomena and notions which are the atoms of the system. Namely, this activity itself is, in fact, the construction created by man. Every atom of a theoretical system and teaching as well as every physical quantity is "constructed" by man himself within certain conditionalities. That is to say, every notion, which is an atom for a great logical system, has been taken from another complex natural system by means of abstraction. Then, a certain construction is created on them by means of reasoning. This construction can be either a regularity that is expressed as a formula, or a more complex theoretical system. This activity of construction is usually carried on "the atoms" that are defined by scientists themselves. However, the relations between these "atoms" are also taken from the infinite truth and nature itself. In fact, we simplify the problem very much. There is no such separation in a real scientific research. A scientist studies a problem and relation as a whole. Namely, the aim in the experiment that he conducts is not at all to take "atoms" from reality, but to take the model of a certain part of reality. That is to say, what is learnt in the experiment is not independently mass or force, but a real and natural process that can express the relation between them. What is the most important is that science is not formed by the effort of one scientist. Every scientist conducts his/her study by being guided by a certain initial model formed due to previous studies, abstractions, and determinations.

What is important in philosophy is the correct determination of the unit, because all numbers can also be gained from the repetition of the unit. The matter emphasized by Leibniz is that thinking processes are also similar to this process. It appears that a same basis or some basic opinions can play an introductory role for all opinions. All other opinions, in turn, can be gained from their combinations. However, what is important is to correctly choose these very basic opinions. No one can start from wherever he/she wants, or more precisely, although he/she starts, he/she cannot go far away. It is necessary to start from a point, where the opinion does not come to a deadlock, and which can be a basis for many other opinions, although not for all opinions. In this sense, the establishment of a philosophical system to some extent reminds us of combinatorics. If a model includes many things only with little basis and with acting from a several principles, it testifies that this model is more optimal.

\section{Works Cited}

Aristotle. Metaphysics, translated with an introduction by Hugh Lawson-Tancred. London, New York: Penguin Books Ltd, 1998. Aristotle. The Basic Works of Aristotle, ed. Richard McKeon. New York: The Modern Library, 2001.

Bible, John. <http://biblehub.com/john/12-24.htm>.

Einstein, Albert. "Considerations Concerning the Fundaments of Theoretical Physics.” Science, LXXXXI (1940): 487-92.

Frege, Gottlob. The Foundations of Arithmetic: A Logico-Mathematical Enquiry Into the Concept of Number. New York: Harper Torchbooks, 1960.

Ibn Arabi, Muhyiddin. Fusus al-Hikam (The Bezels of Wisdom), ed. Abu al-'Ala'. Beirut: Dar Ihya al-kutub al- Arabiyya, 1946.

Plato. Complete Works, with introduction and notes, by John M. Cooper; associate editor, D.S. Hutchinson. Indianapolis, Ind: Hackett Publishing Company Inc., 1997.

Quine, Willard V.O.. Word and Object. Cambridge: MIT Press Ltd., 1960.

Whitehead, Alfred N.. Science and the Modern World. Lowell Lectures 1925. New York: The Macmillan Company, 1925. 